\title{
A Retrospective Review of the Clinical Experience of Linezolid with or Without Rifampicin in Prosthetic Joint Infections Treated with Debridement and Implant Retention
}

\author{
Laura Morata $\cdot$ Eric Senneville $\cdot$ Louis Bernard $\cdot$ Sophie Nguyen • \\ Rodolphe Buzelé · Jérome Druon • Eduard Tornero • Josep Mensa • \\ Alex Soriano \\ To view enhanced content go to www.infectiousdiseases-open.com \\ Received: June 18, 2014 / Published online: August 20, 2014 \\ (C) The Author(s) 2014. This article is published with open access at Springerlink.com
}

\section{ABSTRACT}

Introduction: Debridement and prosthesis retention, combined with a prolonged antibiotic regimen including rifampicin, is an accepted therapeutic approach when the duration of symptoms is less than 4 weeks and there are no radiological signs of loosening. The outcome of patients managed with this strategy has been previously assessed in several articles with success rates of $60-90 \%$. This study aims to

Electronic supplementary material The online version of this article (doi:10.1007/s40121-014-0032-z) contains supplementary material, which is available to authorized users.

L. Morata $(\varangle) \cdot$ E. Tornero · J. Mensa - A. Soriano Bone and Joint Infection Unit, Institut

d'Investigacions Biomèdiques August Pi i Sunyer, Hospital Clínic of Barcelona, C/Villarroel 170, 08036 Barcelona, Spain

e-mail: lmorata@clinic.ub.es

E. Senneville $\cdot$ S. Nguyen

Lille-Tourcoing French Referent Center for Osteoarticular Infection, Lille University Hospital, Lille, France

L. Bernard · R. Buzelé

Infectious Diseases Unit, Tours University Hospital,

Tours Cedex, France

J. Druon

Regional French Referent Center for Osteo-articular Infection, Tours Cedex, France review the clinical experience with linezolid in 3 different hospitals from Spain and France in patients with prosthetic joint infection (PJI) managed with debridement, retention of the implant and treated with linezolid with or without rifampicin.

Methods: Patients with an acute PJI who underwent open debridement with implant retention treated with linezolid for more than 7 days in 3 hospitals from Barcelona, Tours and Lille between 2005 and 2011 were retrospectively reviewed. Relevant information about demographics, co-morbidity, type of implant, surgical treatment, microorganism isolated, antimicrobial therapy, adverse events (AEs) and outcomes were recorded from patients.

Results: A total of 39 patients were retrospectively reviewed. The mean age (SD) was 70.5 (8.8) years and 9 patients had diabetes mellitus (23\%). There were 25 (64\%) knee prostheses, 13 (33\%) hips and 1 shoulder (3\%). The median interquartile range (IQR) days from arthroplasty to infection diagnosis was 17 (19-48) and 33 (85\%) cases were diagnosed within the first 60 days. The median (IQR) duration of antibiotic treatment was 70.5 
(34-96) days and the median (IQR) number of days on linezolid treatment was 44.5 (30-81). AEs were observed in 15 patients (38\%), with gastrointestinal complaints in 8 cases and anemia in 5 being the most frequent. After a median (IQR) follow-up of 2.5 (1.8-3.6) years, there were 11 failures (28\%) (8 relapses and 3 new infections). The failure rate was higher in the rifampicin group (36\% vs. $18 \%$ ) mainly due to a higher relapse rate ( $27 \%$ vs. $12 \%$ ) although differences were not statistically significant.

Conclusion: Management of acute PJIs with debridement and retention of the implant linezolid, with or without rifampicin, is associated with a high remission rate and it is an alternative treatment for infections due to fluoroquinolone and/or rifampicin-resistant staphylococci.

Keywords: Debridement; Infectious diseases; Linezolid; Prosthetic joint infection; Rifampicin; Treatment outcome

\section{INTRODUCTION}

Debridement and prosthesis retention, combined with a prolonged antibiotic regimen including rifampicin, is an accepted therapeutic approach when the duration of symptoms is less than 4 weeks and there are no radiological signs of loosening. The outcome of patients managed with this strategy has been previously assessed in several articles with success rates between $60 \%$ and $90 \%$ [1-6]. The best results have been reported when rifampicin was associated with fluoroquinolones $[4,5]$; however, the rate of multi-resistant staphylococci, including fluoroquinolones, is high and therefore, oral antibiotic alternatives are necessary. Linezolid has a $100 \%$ oral bioavailability and reaches high concentrations in musculoskeletal tissues (skin, synovial fluid and bone) [7-9]; therefore, it is an attractive oral alternative and some data from experimental foreign-body infection model showed good results [10]. Recently, two studies performed in healthy volunteers have analyzed the interaction between linezolid and rifampicin after 3 days of combined therapy $[11,12]$. Both articles support the interaction and found a reduction of about 30\% in the area under the concentration-time curve (AUC) of linezolid. In addition, 2 cases of orthopedic implant infections where this combination was associated with low linezolid serum concentrations and clinical failure have been described [13]. However, the clinical experience with this combination is still scarce.

The aim of the present study was to retrospectively review the clinical experience with linezolid in 3 different hospitals from Spain and France in a particular group of patients with a prosthetic joint infection (PJI), who underwent open debridement with retention of the implant, whilst being treated with linezolid with or without rifampicin.

\section{METHODS}

\section{Study Design}

A retrospective observational study was performed in 3 hospitals from Barcelona, Tours and Lille between 2005 and 2011. All patients included had an acute PJI, were treated with an open debridement with implant retention and received linezolid for more than 7 days. Relevant information about demographics, co-morbidity, type of implant, surgical treatment, microorganism isolated, antimicrobial therapy, adverse events (AEs) and outcomes were recorded. Linezolid dose was $600 \mathrm{mg} / 12 \mathrm{~h}$. When rifampicin was added, 
the dose varied from $600 \mathrm{mg} / 24 \mathrm{~h}$ to $10 \mathrm{mg} / \mathrm{kg} /$ 12 (not exceeding $900 \mathrm{mg} / 12 \mathrm{~h}$ ). In case of polymicrobial infection, ciprofloxacin or a $\beta$-lactam were added according to the Gramnegative antibiogram.

\section{Compliance with Ethics Guidelines}

This study was approved by the Ethics Committee of our institution.

This article does not involve any new studies of human or animal subjects performed by any of the authors.

\section{Definitions}

PJIs were defined by the presence of local inflammation, macroscopic evidence of extension of the infection through the capsule during open debridement, and isolation of significant microorganisms from deep samples. In the case of coagulase-negative staphylococci, $\geq 2$ positive deep samples were required for considering this microorganism a true pathogen. The infection was considered acute when it occurred within 4 weeks after the arthroplasty (early post-operative infection) or after 4 weeks with acute onset of symptoms (late acute infection).

Hematological toxicity was defined as a $>2 \mathrm{~g} / \mathrm{L}$ decrease in the basal hemoglobin concentration without another plausible explanation.

Outcome was classified according to the following definitions: (1) remission, when the patient had no symptoms of infection, the C-reactive protein (CRP) was $<1 \mathrm{mg} / \mathrm{dl}$ and the prosthesis was retained after at least 1 year of follow-up; or (2) failure, when inflammatory signs and high CRP reappear during or after treatment. Failure was divided into relapsed or new infection according to the isolated microorganism. If the isolated microorganism was the same it was considered as relapsed, and when the microorganism was different, it was considered as reinfection. It was not considered failure when the patient developed an aseptic loosening that required the prosthesis to be exchanged and deep samples taken during surgery were negative.

\section{Statistical Analysis}

Categorical variables were described as percentage and continuous variables as median and interquartile range (IQR). Categorical variables were compared by Chisquare test or Fisher's exact test when necessary and continuous variables by Mann-Whitney $U$ test. The Kaplan-Meier survival method was used to estimate the cumulative probability of being in remission in the last visit in those patients receiving or not receiving rifampicin. The Log-Rank test was applied to evaluate the influence of rifampicin. Statistical significance was defined as a two-tailed $P<0.05$. The analysis was performed using SPSS, version 20.0 (SPSS, Inc., Chicago, IL, USA).

\section{RESULTS}

A total of 39 patients were retrospectively reviewed. The mean age (SD) was 70.5 (8.8) years, 21 were females (54\%) and 9 patients had diabetes mellitus (23\%). There were 25 (64\%) knee prostheses, 13 (33\%) hips and 1 shoulder (3\%). Only 4 (10\%) were late acute infections. The median (IQR) days from arthroplasty to infection diagnosis was 17 (19-48) and 33 (85\%) cases were diagnosed within the first 60 days. Infections were monomicrobial in 24 (62\%) cases and polymicrobial in 15 (38\%), and the isolated microorganisms are described in Table 1. The median (IQR) number of days on 
Table 1 Isolated microorganisms in 39 cases of prosthetic joint infection

\begin{tabular}{ll}
\hline Microorganism & Number (\%) \\
\hline Staphylococcus aureus & $9(17)$ \\
Methicillin-susceptible & 4 \\
Methicillin-resistant & 5 \\
Coagulase-negative staphylococci & $33(61)$ \\
Methicillin-susceptible & 10 \\
Methicillin-resistant & 23 \\
Enterococcus faecalis & $4(7)$ \\
Streptococcus viridans & $2(4)$ \\
Enterobacteriaceae $^{\mathrm{b}, \mathrm{c}}$ & $5(9)$ \\
Pseudomonas aeruginosa $^{\mathrm{c}}$ & $1(2)$ \\
\hline
\end{tabular}

${ }^{a}$ The percentage is referred to the total number of isolated microorganisms $(n=54)$ that sum more than 39 because 15 patients had a polymicrobial infection

b Escherichia coli, Klebsiella pneumoniae, Proteus mirabilis, Citrobacter freundii and Enterobacter cloacae

c These microorganisms were always part of a polymicrobial infection with other Gram-positive

linezolid treatment was 44.5 (30-81) and the median (IQR) duration of all antibiotic treatment was 70.5 (34-96) days, including treatment for microorganisms not covered by linezolid in polymicrobial infections. AEs were observed in 15 patients (38\%), with gastrointestinal complaints (nausea, vomiting or diarrhea) in 10 cases and hematological toxicity in 5 cases the most frequent. There were 11 failures (28\%) including 8 (21\%) relapses and 3 new infections (8\%). Therefore, 28 patients $(72 \%)$ were in remission after a median (IQR) follow-up of 2.5 (1.8-3.6) years from stopping antibiotic treatment.

In 22 patients (56\%) rifampicin was given concomitantly with linezolid and 17 did not receive rifampicin because of resistance or drug interaction (i.e., acenocoumarol). The characteristics of patients according to whether or not they received rifampicin are shown in Table 2. Although no difference between both groups was statistically significant, patients receiving rifampicin had a higher rate of diabetes mellitus ( $27 \%$ vs. $18 \%$ ), a longer duration of symptoms before open debridement (9 vs. 2 days), and all MRSA infections were recorded in the rifampicin group (5 vs. 0 ). The remission rate was lower in the rifampicin group ( $64 \%$ vs. $82 \%, P=0.28$ ) due to a higher relapse rate $(27 \%$ vs. $12 \%)$. There were 9 infections due to Staphylococcus aureus, 8 cases (including the 5 MRSA infections in the rifampicin group) were considered in remission (89\%) and 1 patient had a new infection. In contrast, 15 out of 26 infections were due to coagulase-negative staphylococci.

CoNS were in remission (58\%) and 7 (27\%) were due to infection relapse. The cumulative probability of being in remission in the last visit in patients receiving or not rifampicin is shown in Fig. 1 (Log-Rank test, $P=0.25$ ). There were no differences in the total number of AEs between both groups; however, gastrointestinal complains were more frequent in the rifampicin group (32\% vs. $18 \%)$ while hematological toxicity was more frequent in the group without rifampicin (24\% vs. $5 \%)$.

\section{DISCUSSION}

An alternative agent for treating PJIs due to fluoroquinolone-resistant staphylococci is necessary [14]. In the present study, acute PJIs were managed with debridement, retention of the implant and linezolid with a remission rate of $72 \%$ and when considering only relapses (isolation of the same species), it was $80 \%$. These results are similar to those presented by Bassetti et al. [15] using the same surgical strategy and linezolid alone in 20 PJIs with a 
Table 2 Characteristics of patients receiving or not rifampicin concomitantly with linezolid

\begin{tabular}{|c|c|c|c|}
\hline Characteristics & $\begin{array}{l}\text { Receiving } \\
\text { rifampicin }(n=22)\end{array}$ & $\begin{array}{l}\text { Not receiving } \\
\text { rifampicin }(n=17)\end{array}$ & $P$ \\
\hline Median (IQR) age & $71(63-75)$ & $75(66-77)$ & 0.31 \\
\hline Male sex (\%) & $9(41)$ & $9(53)$ & 0.45 \\
\hline Diabetes mellitus (\%) & $6(27)$ & $3(18)$ & 0.37 \\
\hline Type of implant (\%) & & & 0.50 \\
\hline Hip prosthesis & $7(32)$ & $6(35)$ & \\
\hline Knee prosthesis & $15(68)$ & $10(59)$ & \\
\hline Shoulder prosthesis & - & $1(6)$ & \\
\hline Age of prosthesis & $30(21-55)$ & $24(17-32)$ & \\
\hline Late acute infections (\%) & $2(9)$ & $2(12)$ & 1 \\
\hline Median (IQR) days of symptoms before debridement & $9(3-25)$ & $2(1-22)$ & 0.14 \\
\hline Fever $(\%)$ & $3(14)$ & $2(12)$ & 1 \\
\hline Bacteremia (\%) & $2(9)$ & $1(6)$ & 1 \\
\hline Median (IQR) leukocyte count (cells $/ \mathrm{mm}^{3}$ ) & $8,400(6,400-9,600)$ & $6,950(5,750-8,125)$ & 0.18 \\
\hline Median (IQR) C-reactive protein $(\mathrm{mg} / \mathrm{dL})$ & $4(2-11)$ & $3(1-5)$ & 0.22 \\
\hline \multicolumn{4}{|l|}{ Microorganisms } \\
\hline S. aureus (MR) & $6(5)$ & $3(0)$ & \\
\hline CoNS (MR) & $18(13)$ & $15(10)$ & \\
\hline E. faecalis & 3 & 1 & \\
\hline S. viridans & 1 & 1 & \\
\hline Enterobacteriaceae & 2 & 3 & \\
\hline P. aeruginosa & 1 & - & \\
\hline Polymicrobial (\%) & $9(41)$ & $6(35)$ & 0.50 \\
\hline Adverse events & $9(41)$ & $8(47)$ & \\
\hline Gastrointestinal (nausea, vomits or diarrhea) & $7(32)$ & $3(18)^{\mathrm{a}}$ & \\
\hline Hematological toxicity & $1(5)$ & $4(24)$ & \\
\hline Peripheral neuropathy ${ }^{\mathrm{b}}$ & $1(5)$ & $1(6)$ & \\
\hline \multicolumn{4}{|l|}{ Outcome (\%) } \\
\hline Remission & $14(64)$ & $14(82)$ & 0.28 \\
\hline Relapse & $6(27)$ & $2(12)$ & \\
\hline New infection & $2(9)$ & $1(6)$ & \\
\hline
\end{tabular}


Table 2 continued

\begin{tabular}{llll}
\hline Characteristics & $\begin{array}{l}\text { Receiving } \\
\text { rifampicin }(\boldsymbol{n}=\mathbf{2 2})\end{array}$ & $\begin{array}{l}\text { Not receiving } \\
\text { rifampicin }(\boldsymbol{n}=\mathbf{1 7})\end{array}$ \\
\hline $\begin{array}{l}\text { Median (IQR) days of follow-up from stopping } \\
\text { antibiotics to the last visit }\end{array}$ & $730(161-1,219)$ & $812(618-1,362)$ & 0.39 \\
\hline
\end{tabular}

$I Q R$ interquartile range, $M R$ methicillin-resistant

a 2 patients also develop hematological toxicity, therefore, the total number of patients with at least 1 adverse event was 15 $(38 \%)$

b Patients developed peripheral neuropathy during the last few days of the treatment

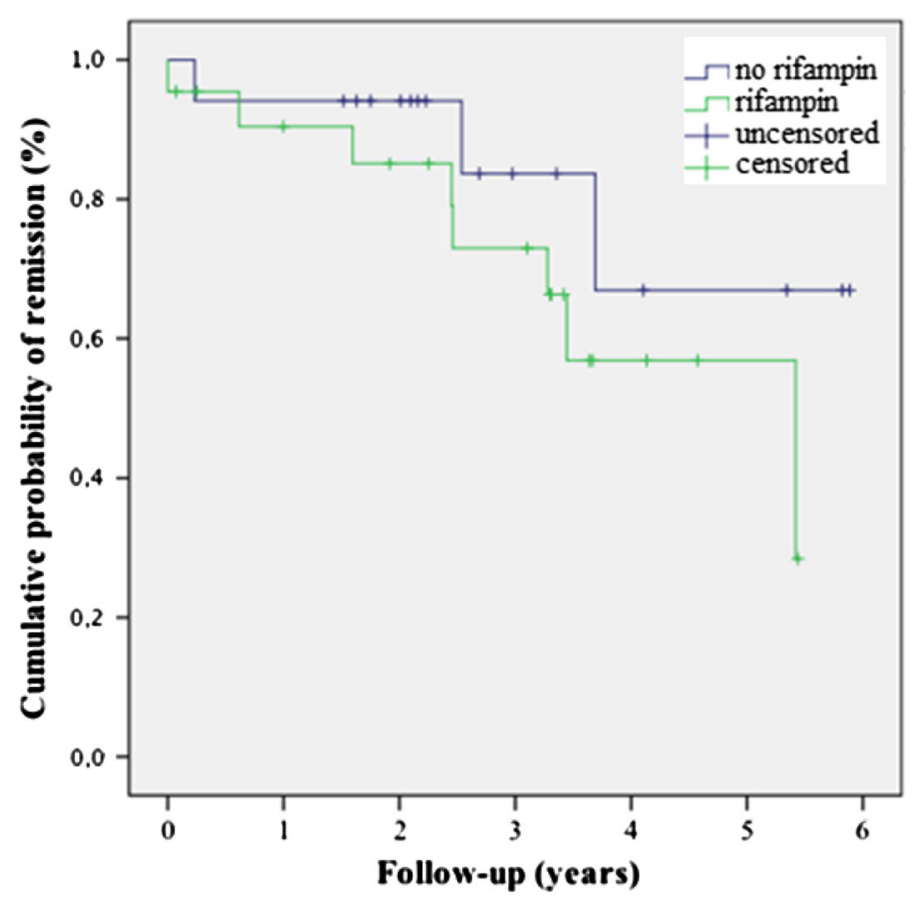

Fig. 1 The cumulative probability of being in remission according to whether the patient received concomitant rifampicin or not (Log-Rank test, $P=0.25$ )

remission rate of $80 \%$ and $20 \%$ of relapsing infections. Monotherapy with linezolid was also evaluated by Rao et al. [16] in 11 cases with a remission rate of $95 \%$. Although the experience is limited, these results are in contrast to the $23 \%$ remission rate described using intravenous vancomycin in MRSA PJI treated with retention of the implant [17] and it suggests that linezolid could be an alternative for infections due to multi-resistant staphylococci.
The addition of rifampicin to linezolid would be reasonable [18, 19], particularly when the foreign-body is not removed, due to the potent activity of rifampicin against biofilm bacteria [4, 20]. It has been demonstrated that rifampicin reduces about 30\% the AUC of linezolid [11, 12]; however, the clinical implication of this interaction is not well established. This combination was assessed in a retrospective study that reviewed 28 osteomyelitis and 
orthopedic implant infections [21]. The success rate was $89.2 \%$, however, only 4 cases were managed without removing the implant. In contrast, Gomez et al. [22] showed a success rate of $69 \%$ but, in this series, all patients were managed with implant retention and rifampicin. In our cohort, no statistically significant difference was observed in the success rate between those patients receiving or not receiving rifampicin but slightly worse results among those receiving rifampicin were observed. This finding could be explained, at least in part, because these patients had a higher rate of diabetes mellitus (32\% vs. $18 \%$ ), and a longer duration of symptoms before open debridement (9 days vs. 2 days), variables that have been associated with a higher failure rates [23]. MRSA infections were all in the group of rifampicin and all achieved remission; therefore, this difference cannot explain the difference between the 2 groups. In addition, it is not possible to rule out a low linezolid concentration in the rifampicin group as an additional explanation. Linezolid is a time-dependent antibiotic [24]; therefore, the pharmacodynamic target is to maintain a trough serum concentration around 2 times over the minimum inhibitory concentration (MIC). Since the $\mathrm{MIC}_{90}$ for Gram-positive staphylococci is $2 \mathrm{mg} / \mathrm{L}$ [25], the optimal trough level will be $4 \mathrm{mg} / \mathrm{L}$, a concentration that also it has been associated with low risk of toxicity [26], which is the major concern when linezolid is administered for prolonged time. These results suggest that monitoring trough serum concentration could be useful for improving the outcome, most especially when linezolid is combined with rifampicin, and for avoiding toxicity in patients that require prolonged treatment [27]. Indeed, hematological toxicity was more frequent in the monotherapy group ( $24 \%$ vs. $5 \%$ ) probably due to the higher linezolid concentrations.
The main drawbacks of this study are the low number of patients, the retrospective design, that clonal relationship between microorganism isolated in primary and relapse episodes was not performed in order to confirm the relapse rate and the fact that linezolid concentrations were not measured; however, the information reported is useful to improve the results in PJIs due to resistant staphylococci.

\section{CONCLUSION}

Acute PJIs managed with debridement and retention of the implant linezolid, with or without rifampicin, are associated with a high remission rate and this is therefore an alternative therapy for infections due to fluoroquinolone and/or rifampicin-resistant staphylococci. However, prolonged linezolid may have important AEs that require close monitoring by infectious diseases physicians.

\section{ACKNOWLEDGMENTS}

Sponsorship for this study was funded by Pfizer (Madrid, Spain) and Fundación Privada Máximo Soriano Jiménez (Barcelona, Spain).

All named authors meet the ICMJE criteria for authorship for this manuscript, take responsibility for the integrity of the work as a whole, and have given final approval for the version to be published.

Conflict of interest. A. Soriano has received honoraria for public speaking and from advisory boards of Pfizer and Novartis.

J. Mensa has received honoraria for public speaking and from advisory boards of Pfizer and Novartis. 
E. Senneville has received honoraria for public speaking and from advisory boards of Sanofi-Aventis, Pfizer and Novartis.

L. Bernard has received honoraria for public speaking and from advisory boards of Pfizer and Astellas.

L. Morata, S. Nguyen, R. Buzele, J. Druon and E. Tornero declare no conflicts of interest.

Compliance with ethics guidelines. This study was approved by the Ethics Committee of our institution.

This article does not involve any new studies of human or animal subjects performed by any of the authors.

Open Access. This article is distributed under the terms of the Creative Commons Attribution Noncommercial License which permits any noncommercial use, distribution, and reproduction in any medium, provided the original author(s) and the source are credited.

\section{REFERENCES}

1. Cobo J, Miguel LGS, Euba G, et al. Early prosthetic joint infection: outcomes with debridement and implant retention followed by antibiotic therapy. Clin Microbiol Infect. 2011;17:1632-7.

2. Vilchez F, Martínez-Pastor JC, Garcia-Ramiro S, et al. Outcome and predictors of treatment failure in early post-surgical prosthetic joint infections due to Staphylococcus aureus treated with debridement. Clin Microbiol Infect. 2011;17:439-44.

3. Zimmerli W, Widmer AF, Blatter M, Frei R, Ochsner PE. Role of rifampin for treatment of orthopedic implant-related staphylococcal infections: a randomized controlled trial. Foreign-Body Infection (FBI) Study Group. JAMA. 1998;279: $1537-41$.

4. Lora-Tamayo J, Murillo O, Iribarren JA, et al. A large multicenter study of methicillin susceptible- and methicillin resistant-Staphylococcus aureus prosthetic joint infections managed with implant retention. Clin Infect Dis. 2012;56:182-94.
5. Senneville E, Joulie D, Legout L, et al. Outcome and predictors of treatment failure in total hip/knee prosthetic joint infections due to Staphylococcus aureus. Clin Infect Dis. 2011;53:334-40.

6. Bernard L, Legout L, Zürcher-Pfund L, et al. Six weeks of antibiotic treatment is sufficient following surgery for septic arthroplasty. J Infect. 2010;61:125-32.

7. Livermore DM. Linezolid in vitro: mechanism and antibacterial spectrum. J Antimicrob Chemother. 2003;51(Suppl 2):ii9-16.

8. MacGowan AP. Pharmacokinetic and pharmacodynamic profile of linezolid in healthy volunteers and patients with Gram-positive infections. J Antimicrob Chemother. 2003; 51(Suppl 2):ii17-25.

9. Kutscha-Lissberg F, Hebler U, Muhr G, Köller M. Linezolid penetration into bone and joint tissues infected with methicillin-resistant staphylococci. Antimicrob Agents Chemother. 2003;47:3964-6.

10. Baldoni D, Haschke M, Rajacic Z, Zimmerli W, Trampuz A. Linezolid alone or combined with rifampin against methicillin-resistant Staphylococcus aureus in experimental foreign-body infection. Antimicrob Agents Chemother. 2009;53:1142-8.

11. Gandelman K, Zhu T, Fahmi OA, et al. Unexpected effect of rifampin on the pharmacokinetics of linezolid. In silico and in vitro approaches to explain its mechanism. J Clin Pharmacol. 2011;51:229-36.

12. Egle $H$, Trittler $R$, Kümmerer $K$, Lemmen SW. Linezolid and rifampin: drug interaction contrary to expectations? Clin Pharmacol Ther. 2005;77: 451-3.

13. Hoyo I, Martínez-Pastor J, Garcia-Ramiro S, et al. Decreased serum linezolid concentrations in two patients receiving linezolid and rifampicin due to bone infections. Scand J Infect Dis. 2012;44:548-50.

14. Tornero E, García-Oltra E, García-Ramiro S, et al. Prosthetic joint infections due to Staphylococcus aureus and coagulase-negative staphylococci. Int J Artif Organs. 2012;35:884-92.

15. Bassetti M, Vitale F, Melica G, et al. Linezolid in the treatment of Gram-positive prosthetic joint infections. J Antimicrob Chemother. 2005;55: 387-90.

16. Rao N, Hamilton CW. Efficacy and safety of linezolid for Gram-positive orthopedic infections: a prospective case series. Diagn Microbiol Infect Dis. 2007;59:173-9. 
17. Bradbury T, Fehring TK, Taunton M, et al. The fate of acute methicillin-resistant Staphylococcus aureus periprosthetic knee infections treated by open debridement and retention of components. J Arthroplasty. 2009;24:101-4.

18. Legout L, Valette M, Dezeque H, et al. Tolerability of prolonged linezolid therapy in bone and joint infection: protective effect of rifampicin on the occurrence of anaemia? J Antimicrob Chemother. 2010;65:2224-30.

19. Soriano A, Gómez J, Gómez L, et al. Efficacy and tolerability of prolonged linezolid therapy in the treatment of orthopedic implant infections. Eur J Clin Microbiol Infect Dis. 2007;26:353-6.

20. Zimmerli W, Frei R, Widmer AF, Rajacic Z. Microbiological tests to predict treatment outcome in experimental device-related infections due to Staphylococcus aureus. J Antimicrob Chemother. 1994;33:959-67.

21. Nguyen S, Pasquet A, Legout L, et al. Efficacy and tolerance of rifampicin-linezolid compared with rifampicin-cotrimoxazole combinations in prolonged oral therapy for bone and joint infections. Clin Microbiol Infect. 2009;15:1163-9.

22. Gómez J, Canovas E, Baños V, et al. Linezolid plus rifampin as a salvage therapy in prosthetic joint infections treated without removing the implant. Antimicrob Agents Chemother. 2011;55:4308-10.

23. Brandt CM, Sistrunk WW, Duffy MC, et al. Staphylococcus aureus prosthetic joint infection treated with debridement and prosthesis retention. Clin Infect Dis. 1997;24:914-9.

24. Craig WA. Basic pharmacodynamics of antibacterials with clinical applications to the use of beta-lactams, glycopeptides, and linezolid. Infect Dis Clin North Am. 2003;17:479-501.

25. Jones RN, Kohno S, Ono Y, Ross JE, Yanagihara K. ZAAPS International Surveillance Program (2007) for linezolid resistance: results from 5591 Grampositive clinical isolates in 23 countries. Diagn Microbiol Infect Dis. 2009;64:191-201.

26. Cattaneo D, Orlando G, Cozzi V, et al. Linezolid plasma concentrations and occurrence of drugrelated hematological toxicity in patients with Gram-positive infections. Int J Antimicrob Agents. 2013;41:586-9.

27. Pea F, Viale P, Cojutti P, Del Pin B, Zamparini E, Furlanut M. Therapeutic drug monitoring may improve safety outcomes of long-term treatment with linezolid in adult patients. J Antimicrob Chemother. 2012;67:2034-42. 